\title{
A Numerical Technique to Estimate Water Depths from Remotely Sensed Water Wave Characteristics
}

\author{
K. M. Fassieh \\ Faculty of Engineering, Cairo University, Giza 12613, Egypt \\ Correspondence should be addressed to K. M. Fassieh; khaledfasseh@yahoo.com
}

Received 16 February 2013; Accepted 5 June 2013

Academic Editors: S. Focardi and S. Ishman

Copyright (C) 2013 K. M. Fassieh. This is an open access article distributed under the Creative Commons Attribution License, which permits unrestricted use, distribution, and reproduction in any medium, provided the original work is properly cited.

\begin{abstract}
This paper describes a numerical technique to estimate water depths from remotely sensed water wave characteristics. Two depth inversion models have been developed based on both linear and nonlinear dispersion relations. A simplified technique to get wave height distribution from remotely sensed water surface elevations is presented. Synthetic input data are generated using a refractiondiffraction numerical model. In intermediate water depths, there is good agreement between actual and estimated depths (relative errors are of order 10\%). It is shown that depth inversion using linear dispersion relation overestimates water depth near shoreline. The nonlinear model is seen to improve the inverted depth by $10 \%$ and could retrieve two-dimensional depth profile.
\end{abstract}

\section{Introduction}

As water waves propagate from deep water to shoreline they undergo strong transformation due to shoaling, refraction, diffraction, reflection, bottom friction, breaking, and others. Most of these phenomena are caused by the bottom bathymetry acting on waves. So it is very important to have an accurate knowledge of the sea bottom topography, particularly in the nearshore area. As bottom topography is continuously evolving over time, it is desirable to have methods for continuous monitoring of these changes.

Early techniques used heavy weight lowered over a ship's side. This technique measures the depth only a single point at a time, and so it has been costly and labor intensive. Nowadays bathymetric measurements come from GPS devices and echosounders mounted beneath or over the side of a boat. These recent methods are also inefficient in terms of time and money in case of surveying large distance on regular bases. Only a relatively small area can be typically surveyed because of the laborious, time-consuming process associated with these practices.

Due high cost associated with traditional methods, there was a need to develop lowcost techniques. One approach is solving the inverse problem, that is, using the remotely sensed surface gravity wave behavior to extract water depths. The theoretical relation between bottom topography and the surface wave characteristics was first modeled mathematically using linear wave theory (e.g., [1]). This relationship is given by the linear wave theory dispersion relation.

In the last decade, some depth inversion techniques have been developed based on remotely sensed measurements of surface elevations and wave number distribution. Most methods still use the linear dispersion relationship to carry out depth inversion. For shallow enough water, nonlinear effects and their influence on wave characteristics cannot be neglected.

Grilli et al. [2] use nonlinear dispersion relation to predict the bottom topography for cases of monochromatic wave propagating over a depth varying in one direction only. They use wave phase and wave height distribution as input to their model.

Leu et al. [3] uses linear dispersion relation to predict the bottom topography in a study area of gentle slope.

Misra et. al. [4] developed one-dimensional algorithm to estimate water depths from synthetic two time-lagged 1D profiles of the surface elevation or velocities using one-dimensional linear shallow-water wave equations and one-dimensional form of the Bossinesqu equations.

Leu and Chang [5] calculated the spatial distribution of wave number spectra using two images from the French 
satellite (SPOT). Then assuming that the frequency of wave does not change and the general dispersion relation between the water depth and the wavelength holds during the wave propagation, they determined the coastal water depths from the spatial variation of the wave spectra. Their method was limited to determining bottom topography over coastal zones where water depths are less than about $12 \mathrm{~m}$.

Catalan [6] performed experimental work along flume with fixed bathymetric profile containing a single bar. Hybrid data set was collected consisting of remotely sensed wave data combined with model-generated wave amplitude profiles. They use nonlinear dispersion relation to retrieve the onedimensional depth profile where the barred portion of the bathymetry was not recovered.

Kennedy et al. [7] presented a technique to reconstruct bathymetry using two snapshots of water surface elevation and velocities as input data. The first snapshot is used to initialize the Boussinesq model to compute wave elevation over a suggested uniform slope bathymetry. The bathymetry is iterated until the best fit is reached with the second snapshot. The difference between computed and measured phase speeds is used as base for updating bathymetry at each iteration.

Flampouris et al. [8] used multibeam echo-sounder data to validate depth inversion using different shallow wave theories.

Yoo et al. [9] compute the bathymetry in and near the surf zone, from spatially varying celerity and breakpoint location data. They used oblique digital video as the initial data source and showed that water depths can be computed within 15\% normalized error.

In this paper, two numerical depth inversion models based on both linear and nonlinear dispersion relations are presented. The proposed models could retrieve two-dimensional depth profiles. Comparison between accuracy of both models in different depth regions is presented. Also the validity ranges of both models are clarified. A simplified technique for processing the two snapshots of remotely sensed surface elevation to get the wave amplitude distributions is presented.

\section{Mathematical Formulation}

As waves propagate onshore, their wave numbers and wave heights change with water depths in the coastal zone. Many forms of dispersion relations that govern the relation between local water depth and wave characteristics were derived. Therefore the two-dimensional distribution of wave characteristics may be used to derive the water depth. Many of previous researches used the linear dispersion relationship to carry out depth inversion. The linear dispersion relation takes the form

$$
\frac{c}{c_{0}}=\tanh (k h)
$$

where $c$ is the local phase speed, $k$ is the wave number, $c_{0}=$ $\sqrt{g k}$ is the deep-water phase speed, and $h$ is the local water depth.
It also takes the form

$$
\omega^{2}=g k \tanh (k h),
$$

where $\omega$ is the wave angular frequency.

But for mildly sloping beaches, shallow water regions, and/or finite wave amplitude, nonlinear effects cannot be neglected. Some researchers proposed empirical dispersion relation which models amplitude dispersion in shallow water, for example, Booij [10] and Hedges [11]. Others derived nonlinear dispersion relations which are valid in intermediate and deep-water regions.

Kirby and Dalrymple [12] proposed an approximate composite dispersion relation. This dispersion relation is an empirical combination of different nonlinear theories dependent upon the relative water depth. The Hedges [11] approximation has been used to model nonlinear effects in shallow water while a third-order Stokes theory was used in intermediate and deep water. The asymptotic behavior of the two takes the following form:

$$
\begin{gathered}
\frac{c}{c_{0}}=\left(1+f_{1} \varepsilon^{2} D\right) \tanh \left(k h+f_{2} \varepsilon\right), \\
f_{1}=\tanh ^{5}(k h), \\
f_{2}=\left[\frac{k h}{\sinh (k h)}\right]^{4}, \\
D=\frac{\cosh (4 k h)+8-2 \tanh ^{2}(k h)}{8 \sinh ^{4}(k h)} .
\end{gathered}
$$

For small-amplitude water wave (3) leads to the well-known linear dispersion relation (1).

2.1. Linear Inversion Algorithms. Two depth inversion models based on both linear and nonlinear dispersion relations are presented in this section. In both models I consider that the only unknown is the depth in the study area and all the required wave characteristics will be measured remotely.

As the water wave propagates from deep water to shallow water its wave period $T$ does not change. Then the linear dispersion relation equation (2) can be solved explicitly in terms of measured wave number $(k)$ and the constant angular frequency $\omega=2 \pi / T$ to calculate the local depth $(h)$ :

$$
h=\frac{1}{k} \tanh ^{-1}\left(\frac{\omega^{2}}{g k}\right) .
$$

2.2. Nonlinear Inversion Algorithms. As nonlinearity cannot be neglected in practical cases, the nonlinear dispersion relation is used. Equations (3)-(6) cannot be solved explicitly to get the predicted depth. Assuming that the spatial distributions of wave number $k(x, y)$ and wave height $H(x, y)$ are remotely sensed, the following algorithm is proposed to solve (3)-(6) implicitly for the predicted depth $h(x, y)$.

(1) Calculate the wave period from the deep-water wave number $k_{0}$. 
In deep water $k h>\pi$; hence substitute in (2)

$$
\begin{gathered}
\omega^{2}=\left(\frac{2 \pi}{T}\right)^{2}=g k_{0} \\
T=\frac{2 \pi}{\sqrt{g k_{0}}} .
\end{gathered}
$$

(2) Calculate first guess for the depth distribution $h_{1}(x$, y) using the linear model:

$$
h_{1}(x, y)=\frac{1}{k(x, y)} \tanh ^{-1}\left(\frac{(2 \pi / T)^{2}}{g k(x, y)}\right) \text {. }
$$

(3) Solve the nonlinear dispersion relation, (3)-(6), at each point in the domain using the Newton Raphson method and the initial guess for $h_{1}(x, y)$ :

$$
h_{n+1}(x, y)=h_{n}(x, y)-\frac{F\left(h_{n}\right)}{F^{\prime}\left(h_{n}\right)} \text {, }
$$

where $n$ iteration number,

$$
\begin{aligned}
F\left(h_{n}\right)=\frac{c}{c_{0}}-\left(1+\tanh ^{5}\left(k h_{n}\right)(k|A|)^{2}\right. \\
\left.\times \frac{\cosh \left(4 k h_{n}\right)+8-2 \tanh ^{2}\left(k h_{n}\right)}{8 \sinh ^{4}\left(k h_{n}\right)}\right) \\
\times \tanh \left(k h_{n}+\left[\frac{k h_{n}}{\sinh \left(k h_{n}\right)}\right]^{4} k|A|\right), \\
F^{\prime}\left(h_{n}\right)=\frac{d}{d h} F\left(h_{n}\right) .
\end{aligned}
$$

(4) Repeat steps 2 and 3 until a sufficiently accurate value is reached.

2.3. Processing Remotely Sensed Data. Remotely sensed measurements of water surface elevations at two or more time intervals are processed to get wave height distribution and wave period as follows.

For two dimensional progressive water wave, consider two snapshots of surface elevation $\eta_{0}$ and $\eta_{1}$ at two time intervals $t=0$ and $t=t_{1}\left(t_{1} \ll\right.$ wave period),

$$
\begin{gathered}
\eta_{0}=\eta(x, y, 0)=\frac{H}{2} \cos (S), \\
\eta_{1}=\eta\left(x, y, t_{1}\right)=\frac{H}{2} \cos \left(S-\omega t_{1}\right),
\end{gathered}
$$

where $H=H(x, y)=$ wave height distribution, $S=S(x, y)=$ phase function distribution, and $\omega=(2 \pi / T)=$ angular frequency.
Hence,

$$
\begin{aligned}
\eta_{1} & =\frac{H}{2} \cos (S) \cos \left(\omega t_{1}\right)+\frac{H}{2} \sin (S) \sin \left(\omega t_{1}\right) \\
& =C 1 \eta_{0}+C 2 \sqrt{\left(\frac{H}{2}\right)^{2}-\eta_{0}^{2},}
\end{aligned}
$$

where $C 1=\cos \left(\omega t_{1}\right)$ and $C 2=\sin \left(\omega t_{1}\right)$.

Hence,

$$
H=H(x, y)=2\left(\left(\frac{\eta_{1}-C 1 \eta_{0}}{C 2}\right)^{2}+\eta_{0}^{2}\right)^{1 / 2} .
$$

\section{Models Validation and Domains of Applicability}

Depth inversion models presented in the previous section need the spatial distributions for wave number and wave height as input. Due to unavailability of actual field data, the inversion models were tested for different bathymetries using synthetic field data generated by numerical modeling. There are many models that can provide the required spatial distribution of wave number and wave amplitude. I use here REF/DIF model [13] which is one of the most famous models in this area and freely available.

To validate the linear and nonlinear depth inversion models, I examine the bathymetry of the large-scale laboratory experiment that was performed in the Long Wave Flume (LWF) at Oregon's O. H. Hinsdale Wave Research Laboratory [14].

The basin is $104 \mathrm{~m}$ long, $3.7 \mathrm{~m}$ wide, and $4.6 \mathrm{~m}$ deep with a programmable flap-type wave maker equipped with active wave. The bottom of the flume was configured into a piecewise continuous, barred profile designed to approximate the bar geometry of an observed field beach profile at a 1:3 reduction in scale and is shown in Figure 1.

Three synthetic data sets (D1, D2, and D3) were generated from progressive monochromatic wave with wave period $T=4 \mathrm{sec}$ and deep-water wave heights $H_{0}(\mathrm{~m})$ are taken to be $0.5 \mathrm{~m}, 0.75 \mathrm{~m}$, and $1 \mathrm{~m}$, respectively. All the three data sets were generated by running on REF/DIF model on LWF bathymetry. These data sets were used in six case studies L1, L2, and L3 are for the linear model while NL1, NL2, and NL3 are for the nonlinear model.

In order to quantify the agreement between the estimated depth $h_{\text {est }}(x)$ and the actual depth $h_{a}(x)$ the percentage relative error $E_{h}(x)$ can be defined as

$$
E_{h}(x)=\frac{h_{\mathrm{est}}(x)-h_{a}(x)}{h_{a}(x)} \times 100 \% .
$$

Also the root mean square error $\varepsilon_{h}$ is calculated for the entire domain as

$$
\varepsilon_{h}=\sqrt{\frac{1}{N} \sum_{i=1}^{N} E_{h}^{2}}
$$

where $N$ is total number of points in the study domain and $i$ is the spatial index.

$E_{h}$ is the relative error at location $i$ computed as in (15). 
Wavemaker

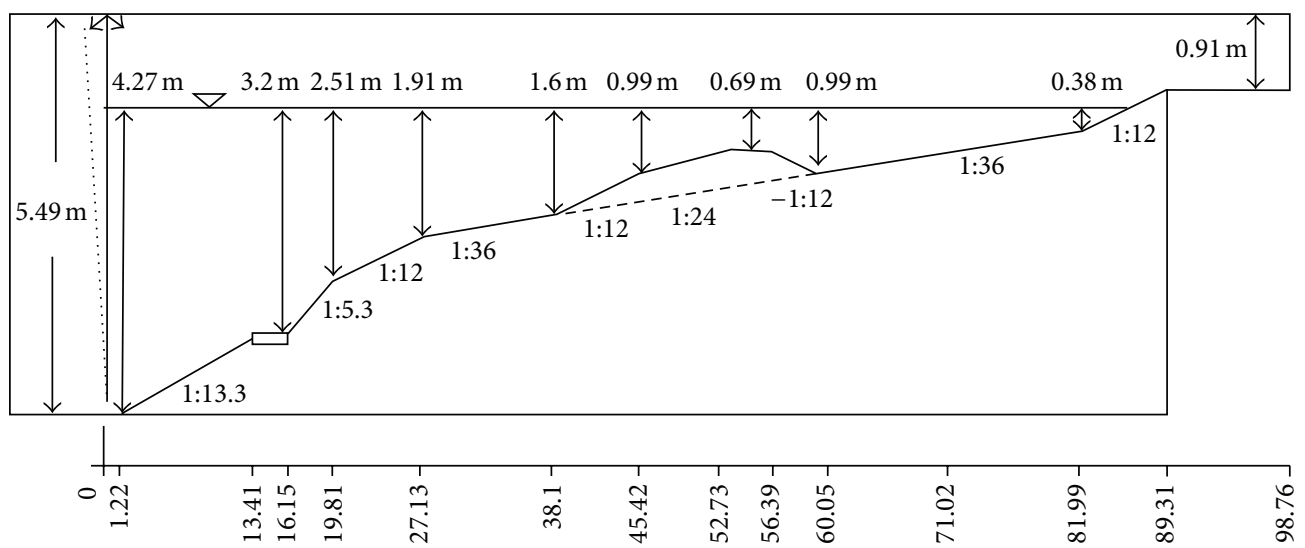

(m)

FIGURE 1: Long wave flume (LWF) bathymetry for Scott et al.s experiment [14].

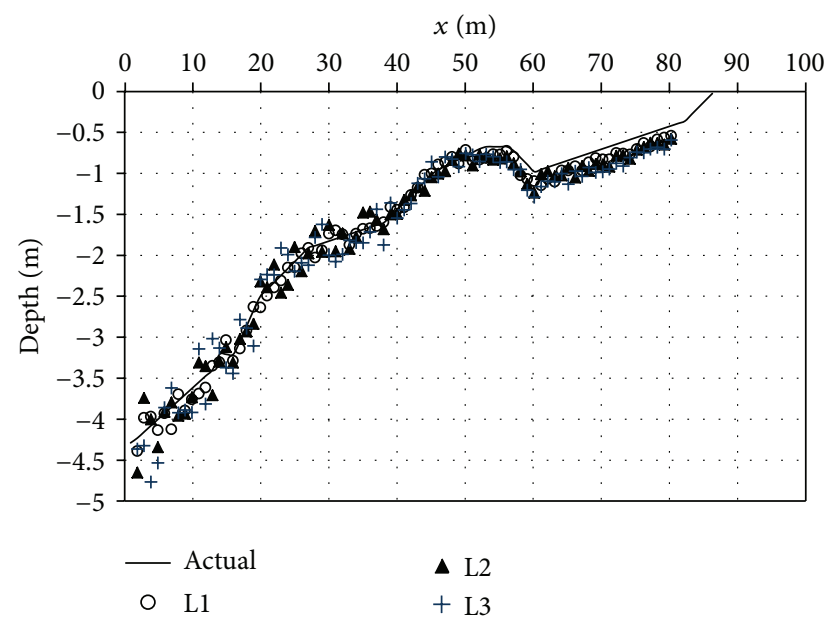

Figure 2: Actual and estimated depths using linear model.

3.1. Linear Model Results. The estimated depths for the linear model test cases are compared with the actual depth in Figure 2. It is shown that the linear model gives good predictions in intermediate water depths. In shallow-water region the estimated depths for the three test cases are overpredicted. It is shown also that the model could not predict the depth correctly in deep water as the wave characteristics become less sensitive to the bottom topography.

Figure 3 shows the percentage relative error for the three case studies. The root mean square errors are $18 \%, 21.6 \%$, and $26.8 \%$ for case studies L1, L2, and L3, respectively. It is shown that error increased when wave heights increased as nonlinear effects could not be neglected.

3.2. Nonlinear Model Results. Figure 4 shows a comparison between actual depth and estimated depths for the nonlinear model test cases NL1, NL2, and NL3. Figure 5 shows the percentage relative error for the three test cases. The root

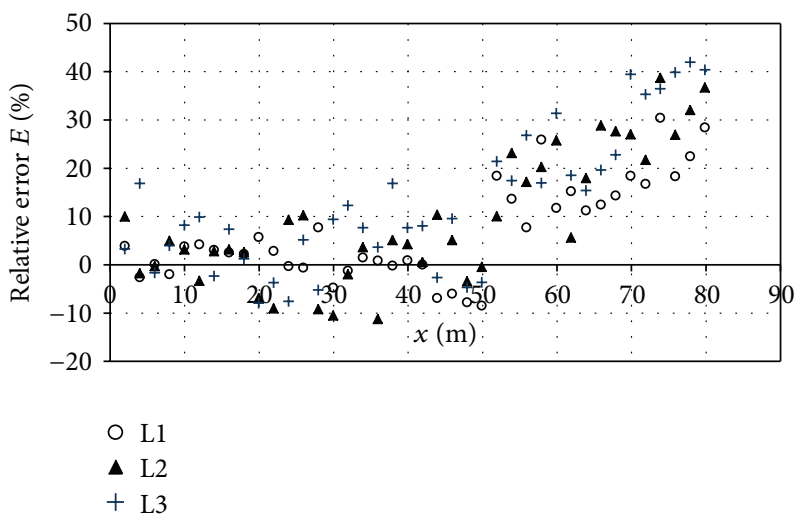

Figure 3: Percentage relative errors for case studies L1, L2, and L3.

mean square errors are $8 \%, 10.5 \%$, and $12.2 \%$ for case studies NL1, NL2, and NL3, respectively.

Comparing results of both linear and nonlinear models, it can be shown that the nonlinear model gives more accurate results than the linear model in the region over and at the lee side of the bar and also in shallow water. The RMS errors reduced from O (20\%) in case of linear model to O (10\%) in case of using nonlinear model. The nonlinear model could not also correctly predict depths in deep-water region. The error in estimating the depth in the region over and at the lee side of the bar may be due to change in wave height by wave reflection.

3.3. Two-Dimensional Case Study. Most of the previous researches were limited to $1 \mathrm{D}$ depth profile inversion. In this section the nonlinear model capabilities in predicting two-dimensional depth profile are presented. The actual depth formula describes a periodic beach with rep channel concentrated at $y=40,120, \ldots \mathrm{m}$ as follows [15]:

$$
h=0.025 x\left[1+20 \exp \left(-3\left(\frac{x}{20}\right)^{1 / 3}\right) \sin ^{10}\left(\frac{\pi y}{80}\right)\right] .
$$




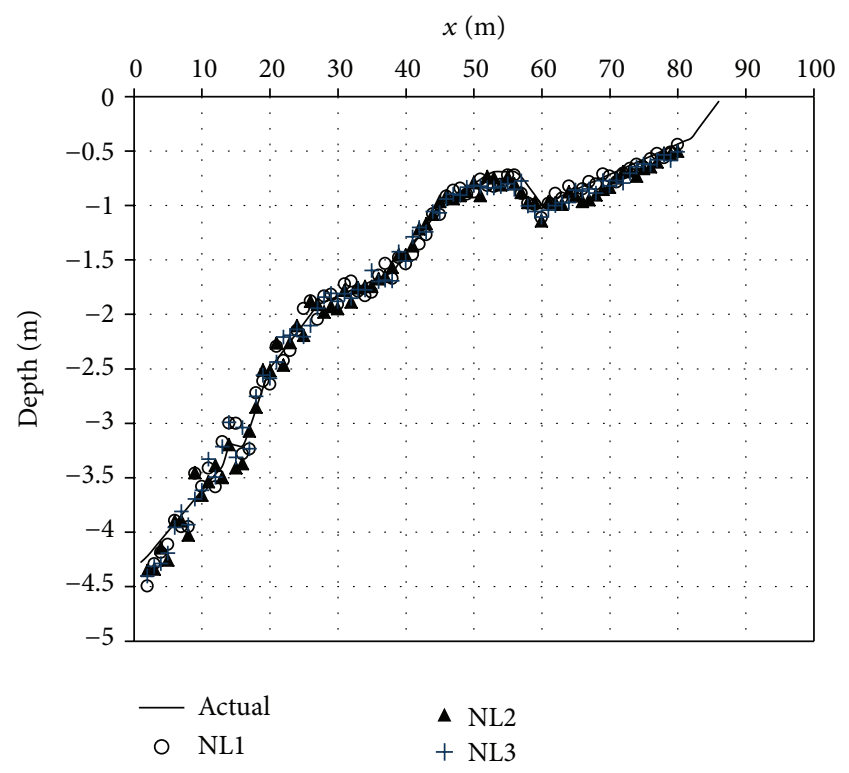

FIgURE 4: Actual and estimated depths using nonlinear model.

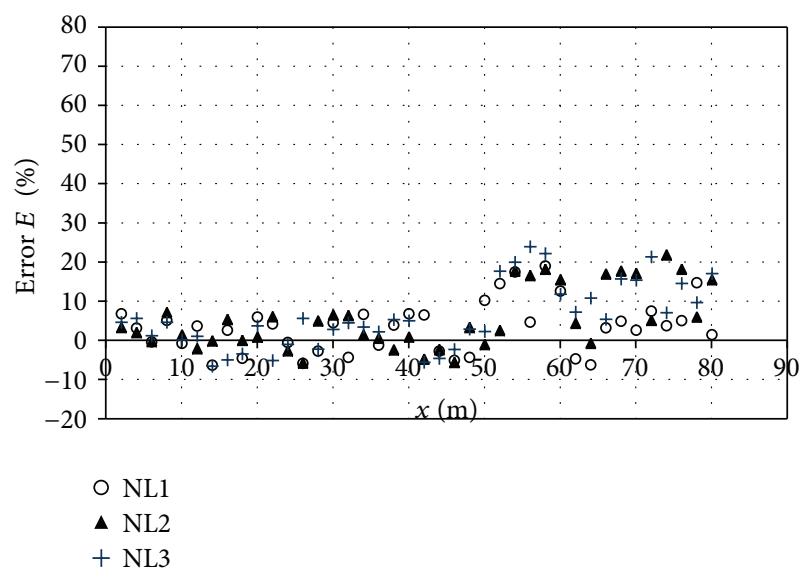

FIGURE 5: Percentage relative errors for case studies NL1, NL2 and NL3.

In this case study, the ability and accuracy of depth inversion technique in case of wave transformation due to refraction, diffraction, and shoaling are checked. As in the first case the input data was generated using an REF/DIF wave transformation model. Deep-water wave height $\left(H_{o}\right)$, wave period $(T)$, and incidence angle $\left(\theta_{o}\right)$ are $1 \mathrm{~m}, 4 \mathrm{sec}$, and $30^{\circ}$, respectively. Figure 6 shows a comparison between the actual and estimated depths at the cross section $(x=40 \mathrm{~m})$. The root mean square errors are $14 \%$.

\section{Conclusions}

(1) The linear model gives good predictions (relative errors are of order 10\%) in intermediate water depths. However the errors increase with shallower depths (relative errors are of order 25\%).

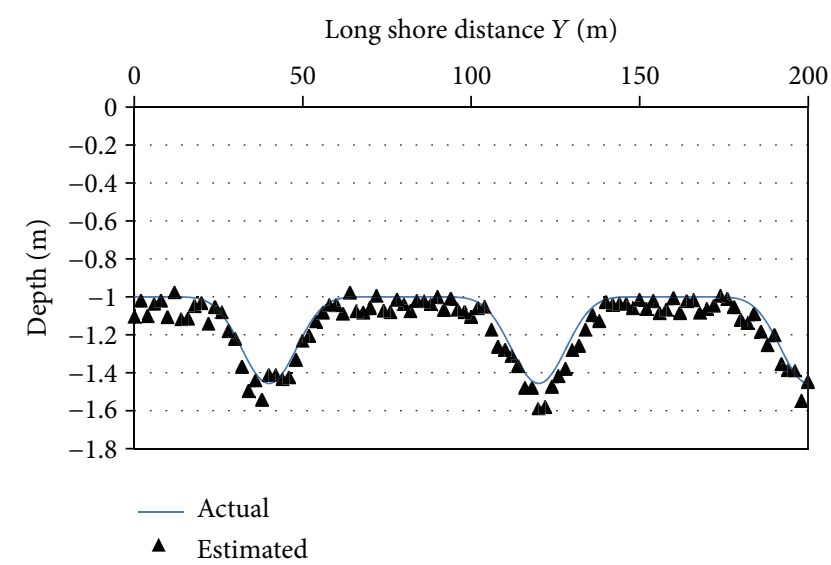

FIGURE 6: Actual and estimated depths at the cross section $(x=$ $40 \mathrm{~m})$.

(2) Estimated depths using linear model are overpredicted in shallow water.

(3) The linear model gives a much poorer depth estimate when increasing the wave height from $0.5 \mathrm{~m}$ to $3.5 \mathrm{~m}$, proving the need for using the nonlinear model.

(4) The RMS errors reduced from O (20\%) in case of linear model to $\mathrm{O}(10 \%)$ in case of using nonlinear model.

(5) Both linear and nonlinear models could not predict the depth correctly in deep water as the wave characteristics become less sensitive to the bottom topography.

(6) The nonlinear model retrieved 2D depth variation with RMS 14\%.

\section{References}

[1] R. G. Dean and R. A. Dalrymple, Water Wave Mechanics for Engineers and Scientists, Prentice-Hall, Englewood Cliffs, NJ, USA, 1984.

[2] S. T. Grilli, M. Asce, and J. Skourup, "Depth inversion for nonlinear waves shoaling over a barred-beach," in Proceedings of the 26th International Conference on Coastal Engineering (ICCE '98), pp. 603-616, June 1998.

[3] L. G. Leu, Y. Y. Kuo, and C. T. Liu, "Coastal bathymetry from the wave spectrum of spot images," Coastal Engineering Journal, vol. 41, no. 1-4, pp. 21-41, 1999.

[4] S. K. Misra, A. B. Kennedy, and J. T. Kirby, "An approach to determining nearshore bathymetry using remotely sensed ocean surface dynamics," Coastal Engineering, vol. 47, no. 3, pp. 265-293, 2003.

[5] L. G. Leu and H. W. Chang, "Remotely sensing in detecting the water depths and bed load of shallow waters and their changes," Ocean Engineering, vol. 32, no. 10, pp. 1174-1198, 2005.

[6] P. Catalan, Hybrid approach to estimating nearshore bathymetry using remote sensing [M.S. thesis], Ocean Engineering, Oregon State University, 2005.

[7] A. B. Kennedy, R. A. Dalrymple, J. T. Kirby, and Q. Chen, "Determination of inverse depths using direct Boussinesq 
modeling," Journal of Waterway, Port, Coastal and Ocean Engineering, vol. 126, no. 4, pp. 206-214, 2000.

[8] S. Flampouris, J. Seemann, and F. Ziemer, "Sharing our experience using wave theories inversion for the determination of the local depth," in Proceedings of the OCEANS 2009-EUROPE, pp. 1-7, Bremen, Germany, May 2009.

[9] J. Yoo, H. M. Fritz, K. A. Haas, P. A. Work, and C. F. Barnes, "Depth inversion in the surf zone with inclusion of wave nonlinearity using video-derived celerity," Journal of Waterway, Port, Coastal and Ocean Engineering, vol. 137, no. 2, pp. 95-106, 2011.

[10] N. Booij, "Gravity waves on water with non-uniform depth and current," Report No. 81-1, Delft University of Technology, 1981.

[11] T. S. Hedges, "An empirical modification to linear wave theory," ICE Proceedings, vol. 61, pp. 575-579, 1976.

[12] J. T. Kirby and R. A. Dalrymple, "An approximate model for nonlinear dispersion in monochromatic wave propagation models," Coastal Engineering, vol. 9, no. 6, pp. 545-561, 1986.

[13] J. T. Kirby, R. A. Dalrymple, and F. Shi, "Combined refraction/diffraction model, REF/DIF 1 version 3.0," Research Report No. CACR-02-02, Center for Applied Coastal Research, Department of Civil and Environmental Engineering, University of Delaware, Newark, NJ, USA, 2002.

[14] C. P. Scott, D. T. Cox, T. B. Maddux, and J. W. Long, "Large-scale laboratory observations of turbulence on a fixed barred beach," Measurement Science and Technology, vol. 16, no. 10, pp. 19031912, 2005.

[15] Edward K. Noda, "Wave-induced nearshore circulation," Journal of Geophysical Research, Oceans and Atmospheres, vol. 79, no. 27, pp. 4097-4106, 1974. 

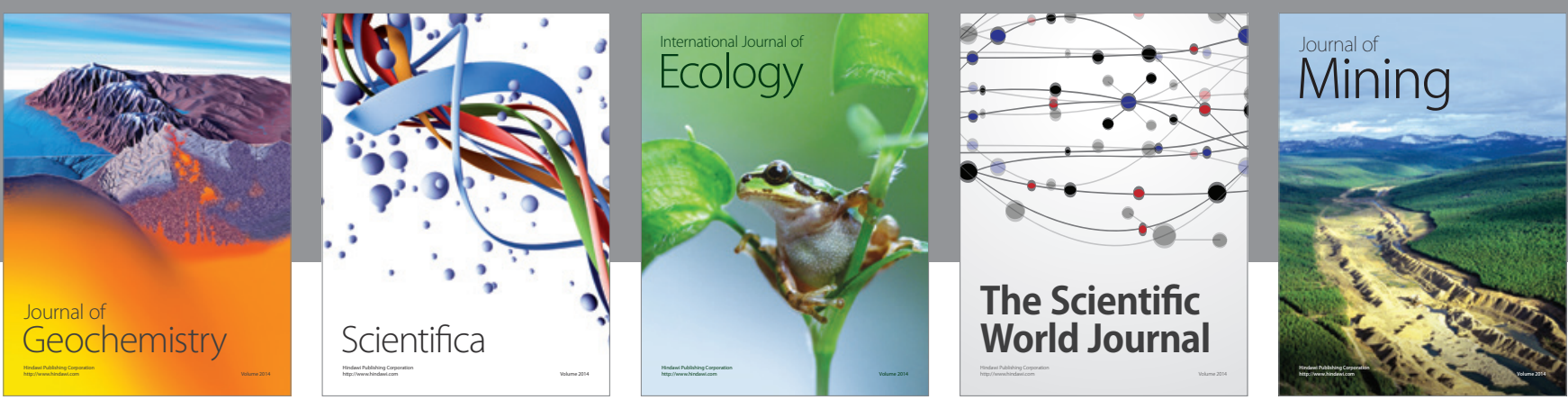

The Scientific World Journal
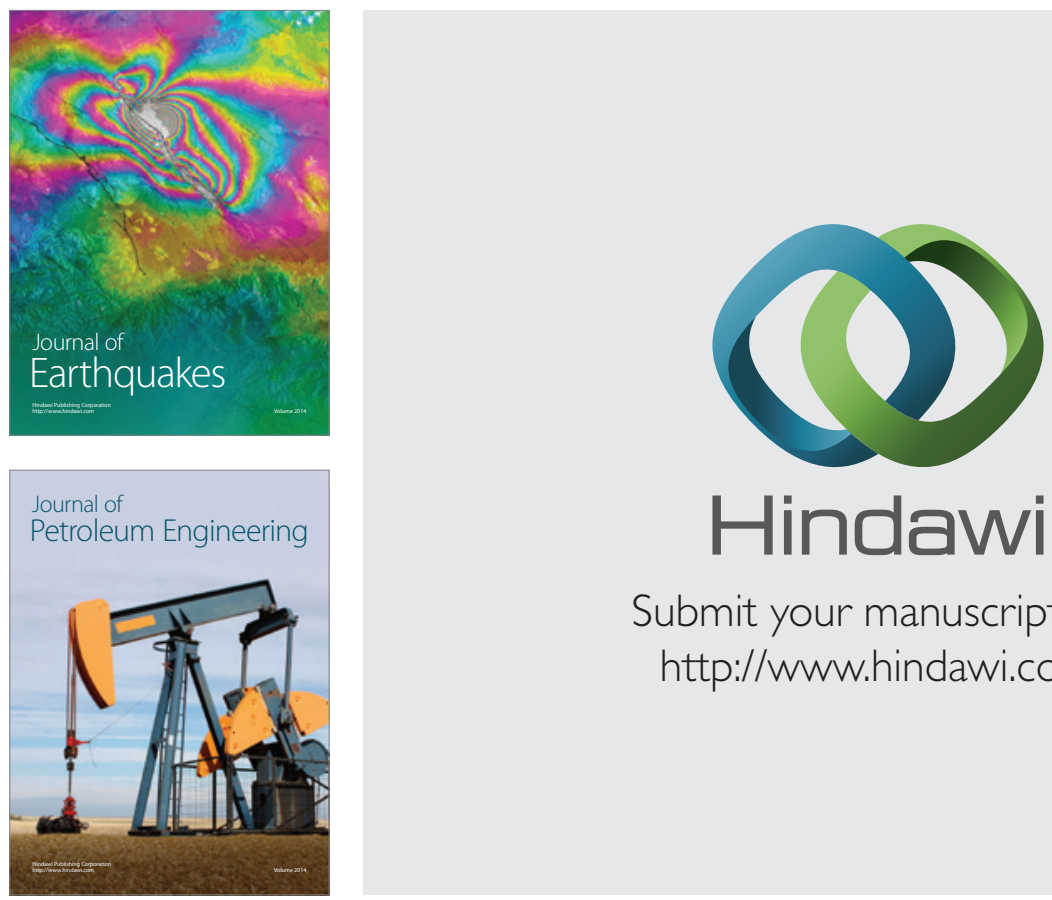

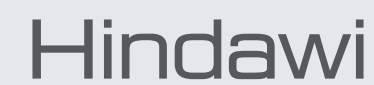

Submit your manuscripts at

http://www.hindawi.com
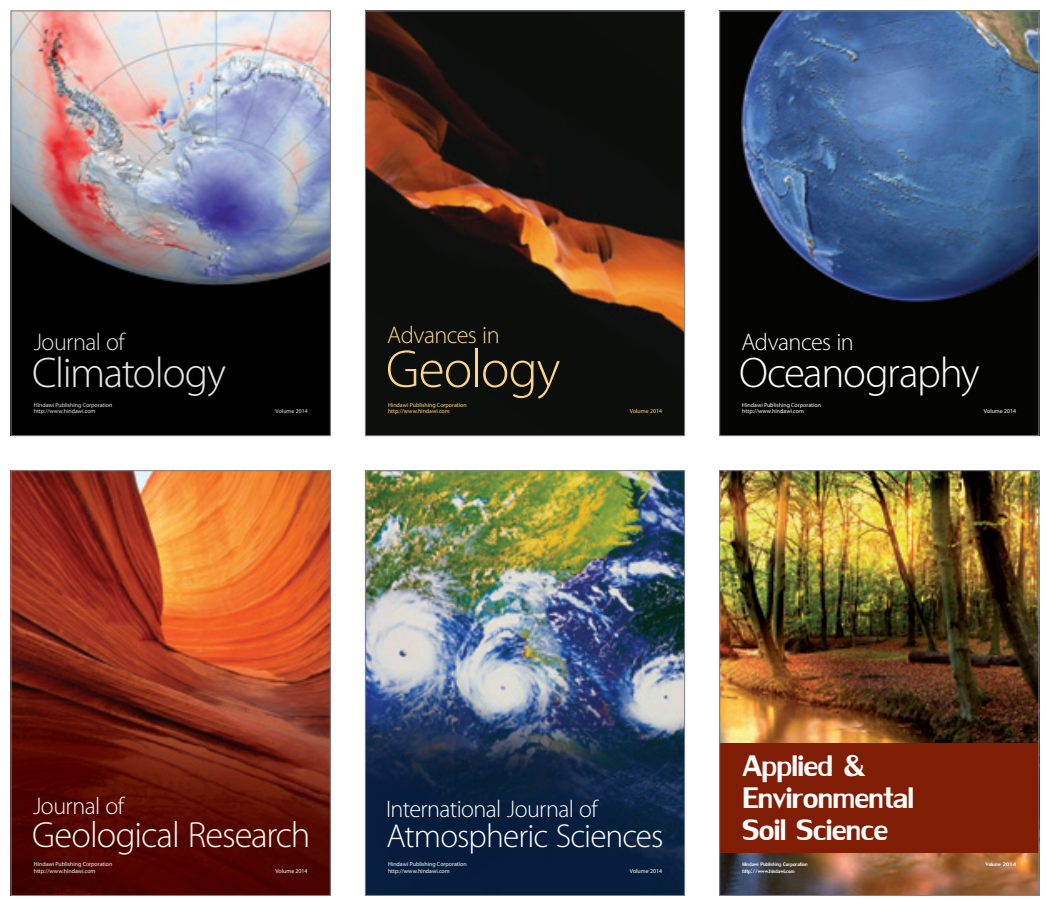
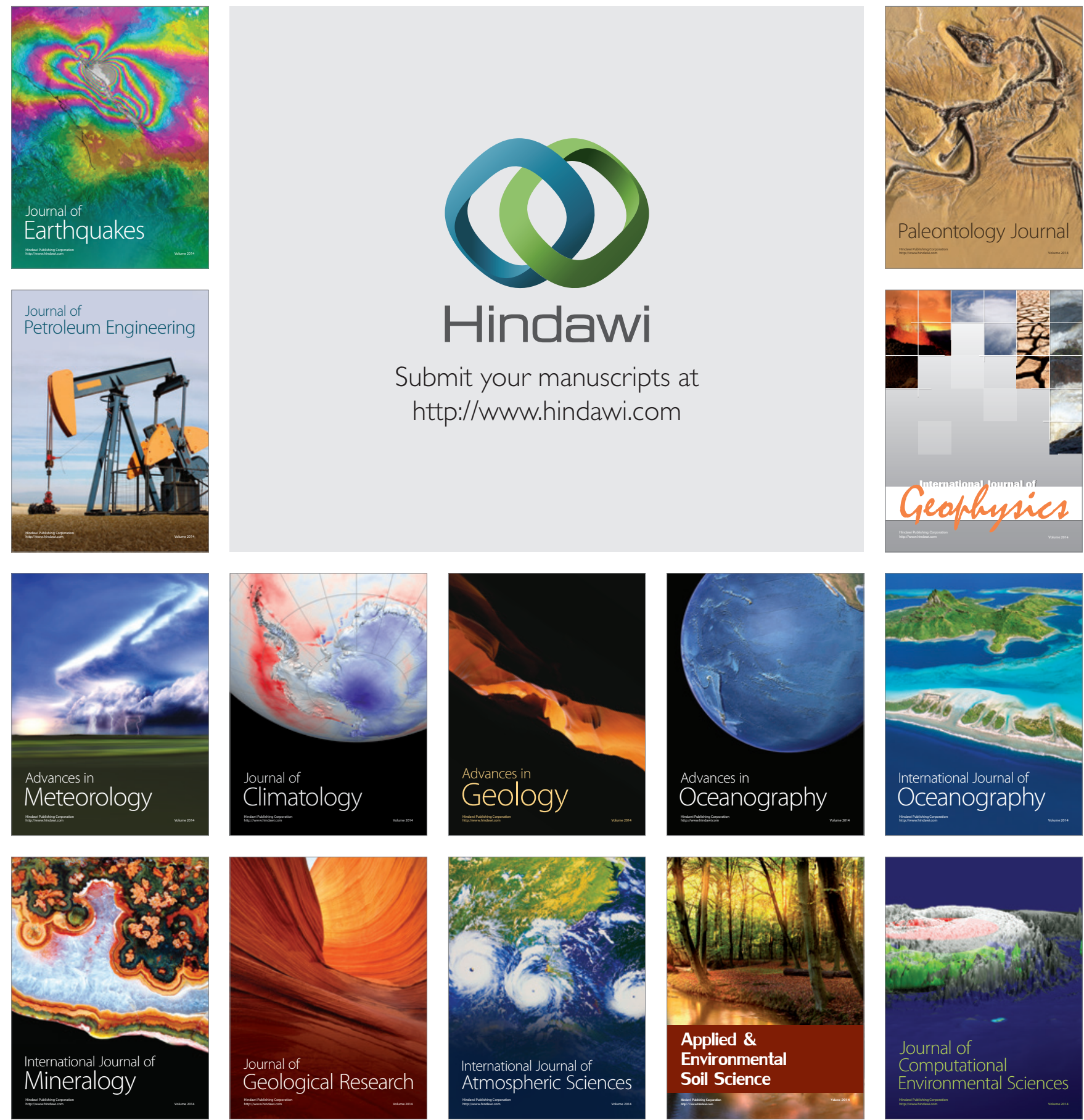